\title{
Further results on common zeros of the solutions of two differential equations
}

Asim Asiri

\section{"Correspondence:}

amkasiri@kau.edu.sa

Department of Mathematics,

Faculty of Science, King Abdulaziz

University, P.O. Box 80203, Jeddah,

21589, Saudi Arabia

\begin{abstract}
Purpose: Two problems are discussed in this paper. In the first problem, we consider one homogeneous and one non-homogeneous differential equations and study when the solutions of these differential equations can have (nearly) the same zeros. In the second problem, we consider two linear second-order differential equations and investigate when the solutions of these differential equations can take the value 0 and a non-zero value at (nearly) the same points.

Method: We apply the Nevanlinna theory and properties of entire solutions of linear differential equations.

Conclusion: In the first problem, the results determine all pairs of such equations having solutions with the same zeros or nearly the same zeros. Regarding the second problem, the results also show all pairs of such equations having solutions taking the value 0 and a non-zero value at (nearly) the same points.
\end{abstract}

Keywords: Nevanlinna theory; differential equations

\section{Introduction}

There has been much research [1-8] on zeros of solutions of linear differential equations with entire coefficients. The principal paper [9] that was published in 1982 by Bank and Laine has stimulated many studies on this kind of problems. The reader is referred to [1012] for background on some applications of the Nevanlinna theory. We use the standard notation of the Nevanlinna theory from [13].

In 1955, Wittich [12] proved the following theorem.

Theorem 1.1 If $f$ is a non-trivial solution of $w^{\prime \prime}+A w=0$, i.e., $f \not \equiv 0$ and $A \not \equiv 0$ is entire, then we have:

(i) $T(r, A)=S(r, f)$.

(ii) Iff has finite order, then $A$ is a polynomial.

(iii) If a is a non-zero complex number, then $f$ takes the value a infinitely often, and in fact, outside a set of finite measure,

$$
N\left(r, \frac{1}{f-a}\right) \backsim T(r, f) .
$$

0 2012 Asiri; licensee Springer. This is an Open Access article distributed under the terms of the Creative Commons Attribution License (http://creativecommons.org/licenses/by/2.0), which permits unrestricted use, distribution, and reproduction in any medium, provided the original work is properly cited. 
The following facts follow from the asymptotic representation for solutions of the equation

$$
w^{\prime \prime}+P w=0
$$

Theorem 1.2 $[9,11]$ Let $P$ be a polynomial of degree $n$, and let $w$ be a non-trivial solution of the equation (1). Then, $w$ has order of growth equal to $\frac{n+2}{2}$. Moreover, if $w$ is a solution of (1) which has infinitely many zeros, then we have

$$
\liminf _{r \rightarrow \infty} \frac{N\left(r, \frac{1}{w}\right)}{r^{(n+2) / 2}}>0 .
$$

Our previous paper [14] studied homogeneous linear differential equations having solutions with nearly the same zeros and proved several results, including the following.

Theorem 1.3 [14] Let $P \not \equiv 0$ be a polynomial of degree $n$. Let $w \not \equiv 0$ be a solution of (1). Assume that $w$ has infinitely many zeros. Suppose that we have a solution $v \neq \equiv 0$ of the differential equation

$$
v^{\prime \prime}+A v=0
$$

such that $A$ is an entire function and $N(r)$ counts zeros of $v$ which are not zeros of $w$ and zeros of $w$ which are not zeros of $v$. Assume that

$$
N(r)+T(r, A)=o\left(r^{(n+2) / 2}\right) .
$$

Then $\frac{v}{w}$ is a constant and $A=P$.

The paper [14] includes further results for homogeneous linear differential equations, and the corresponding problem where $P$ is a transcendental entire function of finite order is studied in [15].

Recently, the same problem, but with non-homogeneous first-order differential equations, has been studied in [16], including the following result.

Theorem 1.4 [16] Assume that $v^{\prime}=A v+B$ and $w^{\prime}=C w+D$, where $A, B, C$ and $D$ are entire functions of order less than 1 and $v, w$ are transcendental functions. Assume that $v=L w$, where L has finitely many zeros and poles, and

$$
T(r, A)+T(r, B)=S(r, v), \quad T(r, C)+T(r, D)=S(r, w) .
$$

Then the following conclusions hold.

(I) If $L$ is a rational function, then $A \equiv C, L$ is a constant and $B=L D$.

(II) If $L$ is a transcendental function, then one of the following cases holds:

(i) $B \equiv D \equiv 0$ and $v, w$ have no zeros.

(ii) $A=-C$ and $B / A, D / C$ are non-zero constants, and

$$
v=c_{1}+c_{2} e^{A_{1}}, \quad w=c_{3}+c_{4} e^{-A_{1}},
$$

where $c_{j} \in \mathbb{C}, A_{1}^{\prime}=A$ and $L=($ constant $) e^{A_{1}}$ 
If, in addition, $L$ has finite order in case (ii), then $A, B, C, D$ are polynomials and so is $A_{1}$.

In this paper, our first result (Theorem 2.1) looks at the same problem but with one homogeneous and one non-homogeneous differential equations. In particular, we consider the first equation to be homogeneous of the second-order with a polynomial coefficient and the second equation to be non-homogeneous of the first-order with entire coefficients.

A further result (Theorem 2.2) studies the case where the solutions of two second-order homogeneous differential equations can take the value 0 and a non-zero value at (nearly) the same points.

\section{Our results}

Our first result is the following theorem.

Theorem 2.1 Suppose that $P \not \equiv 0$ is a polynomial of degree $n$, and $w$ solves (1), and $w \not \equiv 0$ has infinitely many zeros. Suppose that $v \not \equiv 0$ solves

$$
v^{\prime}=A v+B
$$

where $A, B$ are entire and $A B \not \equiv 0$, and

$$
T(r, A)+T(r, B)=o\left(r^{(n+2) / 2}\right) .
$$

Suppose that $L=\frac{w}{v}$ has finitely many zeros and poles (i.e., $w$ and $v$ have the same zeros with finitely many exceptions).

Then $A$ is a polynomial and there exists a polynomial $Q$ such that

$$
w=c_{1} e^{Q} \int e^{-2 Q} d z \quad \text { and } \quad v=c_{2} e^{A_{1}} \int e^{-2 Q} d z
$$

and

$$
P=-\left(Q^{\prime \prime}+Q^{\prime 2}\right) \text { and } B=c_{2} e^{A_{1}-2 Q} \text {, }
$$

where $A_{1}^{\prime}=A$ and $c_{1}, c_{2}$ are constants.

Example 2.1 Take $Q$ to be a polynomial. Let

$$
w=e^{Q} \int_{0}^{z} e^{-2 Q} d z
$$

Then

$$
w^{\prime}=Q^{\prime} w+e^{-Q},
$$

and

$$
w^{\prime \prime}=Q^{\prime \prime} w+Q^{\prime} w^{\prime}-Q^{\prime} e^{-Q}=Q^{\prime \prime} w+Q^{\prime}\left(Q^{\prime} w+e^{-Q}\right)-Q^{\prime} e^{-Q}=\left(Q^{\prime \prime}+Q^{\prime 2}\right) w
$$


So, we have $P=-\left(Q^{\prime \prime}+Q^{\prime 2}\right)$.

Now, let $A_{1}$ be another polynomial, and let

$$
v=e^{A_{1}} \int_{0}^{z} e^{-2 Q} d z
$$

Note that $v$ has the same zeros as $w$. Now, we have

$$
v^{\prime}=A v+e^{A_{1}-2 Q},
$$

where $A=A_{1}^{\prime}$.

We choose $A_{1}$ so that

$$
\operatorname{deg}\left(A_{1}-2 Q\right)<\frac{\operatorname{deg}(P)+2}{2} .
$$

For example, let $A_{1}=2 Q$.

We now state our second result.

Theorem 2.2 Suppose that $P \not \equiv 0$ is a polynomial of degree $n$, and $A$ is an entire function, and suppose that $w$ solves (1) and $v$ solves (3), and $v w \not \equiv 0$. Let $v-1$ and $w$ have, with finitely many exceptions, the same zeros and the same multiplicities. Then one of the following holds.

(A) $w$ has finitely many zeros and $v$ is a polynomial and $A=0$.

(B) $w$ has infinitely many zeros and $P, A$ are non-zero constants and $\frac{v-1}{w}$ is non-constant and

$$
\begin{aligned}
& w=\lambda_{1} e^{\sigma z}+\lambda_{2} e^{-\sigma z}, \\
& v=\lambda_{3} e^{2 \sigma z},
\end{aligned}
$$

where $\sigma, \lambda_{1}, \lambda_{2}, \lambda_{3}$ are non-zero constants.

Example 2.2 If $w=e^{z}-e^{-z}$ and $v=e^{2 z}$, then

$$
v-1=e^{2 z}-1=e^{z}\left(e^{z}-e^{-z}\right)=e^{z} w .
$$

Hence, $v-1$ has the same zeros as $w$. Here $P=-1$ and $A=-4$.

Example 2.3 We give an example to show that the zeros of $v-1$ and $w$ must necessarily have the same multiplicities. To show this, let

$$
w=\sin \frac{z}{2}, \quad v=\cos z .
$$

Then $w=0 \Leftrightarrow \frac{z}{2}=k \pi$, where $k \in \mathbb{Z}$.

Also $v=1 \Leftrightarrow z=k 2 \pi$, where $k \in \mathbb{Z}$.

Therefore, $w$ and $v-1$ have the same zeros but the zeros are simple for $w$, double for $v-1$. Here, $P=\frac{1}{4}$ and $A=1$. 


\section{Proof of Theorem 2.1}

Proof We have

$$
w=L v
$$

So,

$$
w^{\prime \prime}=L^{\prime \prime} v+2 L^{\prime} v^{\prime}+L v^{\prime \prime}
$$

but

$$
v^{\prime \prime}=A^{\prime} v+A v^{\prime}+B^{\prime}=A^{\prime} v+A(A v+B)+B^{\prime}=v\left(A^{2}+A^{\prime}\right)+A B+B^{\prime} .
$$

We also have, using (1), (5), (8), (9) and (10),

$$
\begin{aligned}
0 & =w^{\prime \prime}+P w=L^{\prime \prime} v+2 L^{\prime} v^{\prime}+L v^{\prime \prime}+P L v \\
& =L^{\prime \prime} v+2 L^{\prime}(A v+B)+L\left[v\left(A^{2}+A^{\prime}\right)+A B+B^{\prime}\right]+P L v \\
& =\left[L^{\prime \prime}+2 L^{\prime} A+L\left(A^{2}+A^{\prime}\right)+P L\right] v+2 L^{\prime} B+L\left(A B+B^{\prime}\right) .
\end{aligned}
$$

Let

$$
M=\frac{L^{\prime}}{L}
$$

Then

$$
\frac{L^{\prime \prime}}{L}=M^{\prime}+M^{2}
$$

We divide (11) by $L$, and by using (12) and (13), we get

$$
\begin{aligned}
0 & =\left[\frac{L^{\prime \prime}}{L}+2 \frac{L^{\prime}}{L} A+A^{2}+A^{\prime}+P\right] v+2 \frac{L^{\prime}}{L} B+A B+B^{\prime} \\
& =\left[M^{\prime}+M^{2}+2 M A+A^{2}+A^{\prime}+P\right] v+2 M B+A B+B^{\prime} .
\end{aligned}
$$

The next step is to estimate the growth of $M$.

We know that $\rho(w)=\frac{n+2}{2}$ from [9]. Therefore,

$$
m\left(r, \frac{w^{\prime}}{w}\right)=O(\log r)=o\left(r^{(n+2) / 2}\right)
$$

Claim 3.1 We claim that $T(r, M)=o\left(r^{(n+2) / 2}\right)$.

To show this, we know that $N(r, m)=O(\log r)$ since $M$ has finitely many poles.

Write (5) as

$$
\left(v e^{-A_{1}}\right)^{\prime}=e^{-A_{1}} B,
$$


where $A_{1}^{\prime}=A$.

Then, there exists a constant $c$ such that

$$
v=e^{A_{1}}\left(c+\int_{0}^{z} e^{-A_{1}(t)} B(t) d t\right)
$$

Also, using (6), we can write

$$
\log M(r, A) \leq 3 T(2 r, A)=o\left(r^{(n+2) / 2}\right), \quad M(r, A) \leq \exp \left(o\left(r^{(n+2) / 2}\right)\right) .
$$

Also,

$$
A_{1}(z)=A_{1}(0)+\int_{0}^{z} A(t) d t
$$

So,

$$
M\left(r, A_{1}\right) \leq\left|A_{1}(0)\right|+r M(r, A) \leq O(1)+r \exp \left(o\left(r^{(n+2) / 2}\right)\right) \leq \exp \left(o\left(r^{(n+2) / 2}\right)\right) .
$$

Therefore, we get

$$
M(r, v) \leq \exp \exp \left(o\left(r^{(n+2) / 2}\right)\right), \quad T(r, v) \leq \log M(r, v) \leq \exp \left(o\left(r^{(n+2) / 2}\right)\right) .
$$

We use Lemma 2.3 in [13, p.38] with $R=2 r$ to get

$$
m\left(r, \frac{v^{\prime}}{v}\right)=O(\log r)+O\left(\log ^{+} T(2 r, v)\right) \leq o\left(r^{(n+2) / 2}\right) .
$$

Now, we have $M=\frac{w^{\prime}}{w}-\frac{v^{\prime}}{v}$. So

$$
m(r, M)=o\left(r^{(n+2) / 2}\right)
$$

We also have $N(r, M)=O(\log r)$.

Hence,

$$
T(r, M)=o\left(r^{(n+2) / 2}\right)+O(\log r)=o\left(r^{(n+2) / 2}\right) .
$$

This completes the proof of Claim 3.1.

Using Claim 3.1 and (14), we get

$$
T\left(r, M^{\prime}+M^{2}+2 M A+A^{2}+A^{\prime}+P\right)=o\left(r^{(n+2) / 2}\right)
$$

and

$$
T\left(r, 2 M B+A B+B^{\prime}\right)=o\left(r^{(n+2) / 2}\right) .
$$

Also, by Theorem 1.2, we get

$$
T(r, v) \geq N\left(r, \frac{1}{v}\right)-O(1) \geq(\text { constant }) \cdot r^{(n+2) / 2} .
$$


Therefore, we must have

$$
M_{1}=M^{\prime}+M^{2}+2 M A+A^{2}+A^{\prime}+P \equiv 0
$$

and

$$
M_{2}=2 M B+A B+B^{\prime} \equiv 0
$$

because otherwise we can write $v=-M_{2} / M_{1}$ to get a contradiction.

We now divide (17) by $B$ to get

$$
2 M+A+\frac{B^{\prime}}{B} \equiv 0
$$

So, $B^{\prime} / B$ has finitely many poles, and so $B$ has finitely many zeros. Then we can write $B$ in the form

$$
B=P_{1} e^{P_{2}}
$$

where $P_{1}, P_{2}$ are polynomials.

But then, we can write

$$
B^{\prime} / B=R_{1}
$$

where $R_{1}$ is rational.

Then we also can write

$$
M=-\frac{A}{2}+R_{2},
$$

where $R_{2}$ is rational and $R_{2}=-\frac{1}{2} R_{1}$.

Substitute (18) in (16), we obtain

$$
\begin{aligned}
0 & \equiv\left(-\frac{A^{\prime}}{2}+R_{2}^{\prime}\right)+\left(\frac{A^{2}}{4}-A R_{2}+R_{2}^{2}\right)+\left(-A^{2}+2 A R_{2}\right)+A^{2}+A^{\prime}+P \\
& \equiv\left(\frac{A^{\prime}}{2}+R_{2}^{\prime}\right)+\left(\frac{A^{2}}{4}+A R_{2}+R_{2}^{2}\right)+P \\
& \equiv\left(\frac{A}{2}+R_{2}\right)^{\prime}+\left(\frac{A}{2}+R_{2}\right)^{2}+P .
\end{aligned}
$$

Now, let

$$
N=\frac{A}{2}+R_{2}=\frac{A}{2}-\frac{B^{\prime}}{2 B} .
$$

Also, let

$$
H=e^{\frac{A_{1}}{2}} B^{-\frac{1}{2}} .
$$


So, we get

$$
\frac{H^{\prime}}{H}=\frac{A}{2}-\frac{B^{\prime}}{2 B}=N
$$

Substituting (21) in (19), we obtain

$$
0=N^{\prime}+N^{2}+P=\frac{H^{\prime \prime}}{H}+P, \quad H^{\prime \prime}+P H=0 .
$$

Thus, $\rho(H)<\infty, H$ is entire, and $B$ has no zeros.

Then,

$$
m\left(r, \frac{H^{\prime}}{H}\right)=O(\log r)
$$

Therefore, $A$ is a polynomial.

Since $B$ has no zeros, from (20) we can write

$$
H=e^{Q},
$$

where $Q$ is a polynomial.

Since $w$ and $H$ solve the same equation and are linearly independent (because $w$ has zeros but $H$ does not), we can write

$$
\left(\frac{w}{H}\right)^{\prime}=\frac{\text { (constant) }}{H^{2}} .
$$

Therefore,

$$
w=c_{1} e^{Q} \int e^{-2 Q} d z
$$

where $c_{1}$ is a constant and $Q$ is a polynomial.

Now, we have

$$
\frac{L^{\prime}}{L}=M=-\frac{A}{2}+R_{2}=N-A=\frac{H^{\prime}}{H}-A .
$$

So, we can write

$$
L=(\text { constant }) \cdot H e^{-A_{1}} \text {. }
$$

Therefore, we have

$$
v=\frac{w}{L}=\frac{(\text { constant }) \cdot H \int H^{-2} d z}{H e^{-A_{1}}} .
$$

Hence, using (22), we obtain

$$
v=c_{2} e^{A_{1}} \int e^{-2 Q} d z
$$


where $c_{2}$ is a constant and $A_{1}^{\prime}=A$.

Now, from (23) and (24), we notice that $w$ and $v$ have the same zeros.

Also, differentiating (24), using (22), we have

$$
v^{\prime}=c_{2} A e^{A_{1}} \int H^{-2} d z+c_{2} e^{A_{1}} H^{-2}=A v+c_{2} e^{A_{1}} H^{-2}=A v+c_{2} e^{A_{1}-2 Q} .
$$

Comparing this with (5), we get

$$
B=c_{2} e^{A_{1}-2 Q} \text {. }
$$

Moreover, $H=e^{Q}$ solves $H^{\prime \prime}+P H=0$, and so

$$
-P=\frac{H^{\prime \prime}}{H}=Q^{\prime \prime}+Q^{\prime 2}
$$

This completes the proof of Theorem 2.1.

\section{A lemma needed to prove Theorem 2.2}

In order to prove Theorem 2.2, we must state and prove the following lemma. We include a proof for completeness.

Lemma 4.1 Let $P_{1}, \ldots, P_{n} \in \mathbb{C}$ be distinct, and let $A_{1}, \ldots, A_{n}$ be rational functions such that

$$
1 \equiv A_{1}(z) e^{P_{1} z}+\cdots+A_{n}(z) e^{P_{n} z}
$$

Then there exists $k \in\{1, \ldots, n\}$ such that $P_{k}=0$ and $A_{k}=1$, and $A_{j}=0$ for $j \neq k$.

Proof The proof is by induction. It is obvious that the lemma is true when $n=1$.

Assume that the lemma is true for $m \leq n-1$. Differentiating (25), we get

$$
0 \equiv B_{1}(z) e^{P_{1} z}+\cdots+B_{n}(z) e^{P_{n} z}, \quad B_{j}=A_{j}^{\prime}+P_{j} A_{j} .
$$

Now, we have two cases to consider.

Case (1): Suppose there exists $k$ such that $B_{k} \not \equiv 0$. Without loss of generality, let $k=1$, then we can write

$$
0=1+\frac{B_{2}}{B_{1}} e^{\left(P_{2}-P_{1}\right) z}+\cdots+\frac{B_{n}}{B_{1}} e^{\left(P_{n}-P_{1}\right) z} .
$$

Since we assumed the lemma is true for $m \leq n-1$, there exists $j \in\{2, \ldots, n\}$ such that $P_{j}-P_{1}=0$. But this contradicts our assumption that $P_{1}, \ldots, P_{n}$ are distinct.

Case (2): Suppose that $B_{j}=0$ for each $j$, i.e.,

$$
A_{j}^{\prime}+P_{j} A_{j} \equiv 0
$$

If $P_{j} \neq 0$, then $A_{j} \equiv 0$ because otherwise we have

$$
\frac{A_{j}^{\prime}}{A_{j}}+P_{j} \equiv 0, \quad A_{j}(z) e^{P_{j} z}=c \in \mathbb{C} \backslash\{0\}
$$


But this contradicts the fact that $P_{j} \neq 0$.

So, we have $A_{j} \equiv 0$ for $P_{j} \neq 0$. Thus, (25) becomes (for some $k$ )

$$
1=A_{k}(z) e^{0 z}=A_{k}(z)
$$

and $P_{k}=0$ and $A_{k}=1$.

\section{Proof of Theorem 2.2}

We first note that, outside a set of finite measure, by Theorem 1.1,

$$
T(r, v) \sim N\left(r, \frac{1}{v-1}\right) \leq N\left(r, \frac{1}{w}\right)+O(\log r) \leq O\left(r^{(n+2) / 2}\right)+O(\log r) .
$$

In particular, if $w$ has finitely many zeros, then $v$ is a polynomial, which gives $A=0$. This completes the proof of part (A) in the conclusion.

Assume henceforth that $w$ has infinitely many zeros. Then (26) implies that $\rho(v) \leq(n+$ 2 ) $/ 2$, and so $A$ is a polynomial of degree at most $n$ by the Wiman-Valiron theory [17]. Also, $A \not \equiv 0$ since $v-1$ has infinitely many zeros.

Now, two cases have to be considered.

Case (I). Assume that $P$ is a non-zero constant; then $n=0$ and $A$ is constant. Therefore, we can write

$$
w=c_{1} e^{\alpha z}+c_{2} e^{-\alpha z}, \quad v=d_{1} e^{\beta z}+d_{2} e^{-\beta z},
$$

where $\alpha, \beta \in \mathbb{C} \backslash\{0\}, c_{j}, d_{j} \in \mathbb{C}$ and $c_{j} \neq 0(j=1,2)$.

Since $w$ and $v-1$ have the same zeros with finitely many exceptions, we can write

$$
v-1=R_{1} e^{P_{1}} w,
$$

where $R_{1}$ is a rational function and $P_{1}$ is a polynomial. We know that $\operatorname{deg}\left(P_{1}\right) \leq 1$ because $\rho(w), \rho(v) \leq 1$. We can now write

$$
d_{1} e^{\beta z}+d_{2} e^{-\beta z}-1=R_{1} e^{\gamma z}\left(c_{1} e^{\alpha z}+c_{2} e^{-\alpha z}\right)
$$

where $\gamma \in \mathbb{C}$, and so

$$
1=d_{1} e^{\beta z}+d_{2} e^{-\beta z}-c_{1} R_{1} e^{(\gamma+\alpha) z}-c_{2} R_{1} e^{(\gamma-\alpha) z} .
$$

Now, by using Lemma 4.1, $R_{1}$ is constant and so we can write (28) as

$$
\frac{v-1}{w}=e^{\gamma z+\delta}
$$

where $\delta$ is constant.

Therefore,

$$
d_{1} e^{\beta z}+d_{2} e^{-\beta z}-1=\left(e^{\gamma z+\delta}\right)\left(c_{1} e^{\alpha z}+c_{2} e^{-\alpha z}\right)=c_{1} e^{(\alpha+\gamma) z+\delta}+c_{2} e^{(-\alpha+\gamma) z+\delta} .
$$


Now, by using Lemma 4.1, we get

$$
\alpha+\gamma=\beta,-\beta \text { or } 0, \quad-\alpha+\gamma=\beta,-\beta \text { or } 0,
$$

and $\alpha+\gamma,-\alpha+\gamma, \beta,-\beta, 0$ cannot all be different.

We must now try six cases:

I(a): If $\alpha+\gamma=\beta$ and $-\alpha+\gamma=-\beta$, then $\gamma=0$ and $\alpha=\beta$. But this contradicts (30). Thus, this case cannot happen.

I(b): If $\alpha+\gamma=\beta$ and $-\alpha+\gamma=0$, then $\beta=2 \gamma$ and $\alpha=\gamma$. Substituting these in (30) gives

$$
d_{1} e^{2 \gamma z}+d_{2} e^{-2 \gamma z}-1=h_{1} e^{2 \gamma z}+h_{2}
$$

where $h_{1}, h_{2}$ are constants, which yields $d_{2}=0$. Putting this in (27) gives (7) with $\sigma=\gamma$.

There are four more cases:

$\mathrm{I}(\mathrm{c}): \alpha+\gamma=-\beta$ and $-\alpha+\gamma=\beta$.

$\mathrm{I}(\mathrm{d}): \alpha+\gamma=-\beta$ and $-\alpha+\gamma=0$.

$\mathrm{I}(\mathrm{e}): \alpha+\gamma=0$ and $-\alpha+\gamma=\beta$.

$\mathrm{I}(\mathrm{f}): \alpha+\gamma=0$ and $-\alpha+\gamma=-\beta$.

It is easy to check that case $\mathrm{I}(\mathrm{c})$ is impossible and cases $\mathrm{I}(\mathrm{d}), \mathrm{I}(\mathrm{e}), \mathrm{I}(\mathrm{f})$ all lead to (7) with $\sigma=\gamma$.

From these cases, we find that $\gamma \neq 0$, and so $\frac{\nu-1}{w}=e^{\gamma z+\delta}$ is non-constant. Also, we have (7) and case (B) of the conclusion.

Case (II). Suppose that $P$ is non-constant. We will show that this leads to a contradiction. Let

$$
\frac{v-1}{w}=M=L e^{Q}
$$

where $L$ is a rational function and $Q$ is an entire function.

From (26), we have $\rho(v)<\infty$, and so $Q$ is a polynomial.

Also, from (31), we have

$$
\begin{aligned}
& v=M w+1, \quad v^{\prime}=M^{\prime} w+M w^{\prime}, \\
& v^{\prime \prime}=M^{\prime \prime} w+2 M^{\prime} w^{\prime}+M w^{\prime \prime}=2 M^{\prime} w^{\prime}+w\left(M^{\prime \prime}-P M\right)=-A v=-A(M w+1) .
\end{aligned}
$$

So,

$$
2 M^{\prime} w^{\prime}+\left(M^{\prime \prime}+A M-P M\right) w=-A .
$$

Now, we have two cases to consider.

Case (i): If $M$ is constant, then either $A=P$ and $A=0$, so that $P=0$, which is a contradiction, or

$$
w=\frac{-A}{A M-P M}
$$

is a rational function, which is a contradiction since $w$ has infinitely many zeros. 
Case (ii): If $M$ is non-constant, then $M^{\prime} \not \equiv 0$. Therefore,

$$
w^{\prime}+\left(\frac{M^{\prime \prime}}{2 M^{\prime}}+\frac{(A-P) M}{2 M^{\prime}}\right) w=\frac{-A}{2 M^{\prime}}
$$

where $\frac{M^{\prime \prime}}{2 M^{\prime}}+\frac{(A-P) M}{2 M^{\prime}}$ is rational because $\frac{M^{\prime}}{M}=\frac{L^{\prime}}{L}+Q^{\prime}$ is rational and $\frac{M^{\prime \prime}}{M}$ is rational, and so $\frac{M^{\prime \prime}}{M^{\prime}}=\frac{M^{\prime \prime}}{M} / \frac{M^{\prime}}{M}$ is rational.

Also,

$$
\frac{-A}{2 M^{\prime}}=\frac{-A}{2\left(L^{\prime}+Q^{\prime} L\right) e^{Q}} .
$$

Then we can write (33) as

$$
w^{\prime}=R w+S e^{-Q}
$$

where

$$
R=\frac{P M-A M-M^{\prime \prime}}{2 M^{\prime}}, \quad S=\frac{-A}{2\left(L^{\prime}+Q^{\prime} L\right)}
$$

are rational functions and $Q$ is a polynomial.

Let $U=S e^{-Q}$, then we can write (34) as

$$
w^{\prime}=R w+U
$$

Now, we have two cases to consider:

Case ii(a): If $R \equiv 0$ in (35), then (34) gives

$$
w^{\prime}=S e^{-Q}, \quad w=\frac{-w^{\prime \prime}}{P}=\frac{-\left(S^{\prime}-Q^{\prime} S\right)}{P} e^{-Q},
$$

which is a contradiction since $w$ has infinitely many zeros.

Case ii(b): Assume that $R \not \equiv 0$ in (35); then (35) gives

$$
w^{\prime \prime}=R w^{\prime}+R^{\prime} w+U^{\prime} .
$$

Now, (1) and (35) give

$$
-P w=R(R w+U)+R^{\prime} w+U^{\prime},
$$

and so

$$
\left(R^{\prime}+R^{2}+P\right) w+R U+U^{\prime}=0
$$

Therefore,

$$
R^{\prime}+R^{2}+P \equiv 0,
$$


because if not, $w$ has finitely many zeros, a contradiction. Also,

$$
R U+U^{\prime} \equiv 0 .
$$

Put

$$
G=\frac{1}{U}=T e^{Q},
$$

where $T=1 / S$ is a rational function.

Then,

$$
R=\frac{-U^{\prime}}{U}=\frac{G^{\prime}}{G} .
$$

From (36), we get

$$
0=R^{\prime}+R^{2}+P=\frac{G^{\prime \prime}}{G}+P, \quad G^{\prime \prime}+P G=0 .
$$

So, $G$ solves (1), and since $P \not \equiv 0$ and is a polynomial of degree $n$, we see that $G$ is a transcendental entire function with finitely many zeros and has order $(n+2) / 2$.

Since $w$ and $G$ solve the same equation but $w$ has infinitely many zeros and $G$ has finitely many zeros, $w$ and $G$ are linearly independent, and we can write

$$
w^{\prime} G-w G^{\prime}=c,
$$

where $c$ is a non-zero constant. So,

$$
\left(\frac{w}{G}\right)^{\prime}=\frac{c}{G^{2}} .
$$

By integrating, we get

$$
w=G \int^{z} \frac{c}{G^{2}} d \zeta .
$$

Also, using (31), (38) and (39),

$$
v=1+M w=1+L e^{Q} T e^{Q} \int^{z} \frac{c}{G^{2}} d \zeta=1+H G^{2} \int^{z} \frac{c}{G^{2}} d \zeta,
$$

where $H=L / T$ is a rational function.

Now, we can assume that $c=1$ because if $c \neq 1$, we can multiply $w$ by $1 / c$.

We differentiate (40) to get

$$
v^{\prime}=H^{\prime} G^{2} \int^{z} \frac{1}{G^{2}} d \zeta+2 H G G^{\prime} \int^{z} \frac{1}{G^{2}} d \zeta+H=H+K(v-1),
$$

where

$$
K=\frac{H^{\prime}}{H}+2 \frac{G^{\prime}}{G}
$$

is a rational function. 
So, from (3) and (41), we get

$$
-A v=v^{\prime \prime}=H^{\prime}+K^{\prime}(v-1)+K[H+K(v-1)],
$$

and so

$$
0=v\left(K^{\prime}+K^{2}+A\right)+\left(H^{\prime}-K^{\prime}+K H-K^{2}\right)=v U_{1}+U_{2},
$$

where $U_{1}=K^{\prime}+K^{2}+A$ and $U_{2}=H^{\prime}-K^{\prime}+K H-K^{2}$.

Since $v$ is transcendental and $U_{1}, U_{2}$ are rational functions, we must have

$$
U_{1}=K^{\prime}+K^{2}+A=0
$$

and

$$
U_{2}=H^{\prime}-K^{\prime}+K H-K^{2}=0 .
$$

Claim 5.1 We claim that $H \equiv K$.

To show this, let $H \not \equiv K$.

From (44), we have

$$
\frac{H^{\prime}-K^{\prime}}{H-K}+K=0
$$

From (42), we get

$$
\frac{H^{\prime}-K^{\prime}}{H-K}=-K=\frac{-H^{\prime}}{H}-2 \frac{G^{\prime}}{G} \text {. }
$$

We integrate to get

$$
H-K=\frac{a}{H G^{2}}, \quad G^{2}=\frac{a}{H(H-K)},
$$

where $a$ is a constant. But this contradicts the fact that $H$ and $K$ are rational functions and $G$ is a transcendental function. This completes the proof of Claim 5.1.

Once we have Claim 5.1, (41) gives

$$
v^{\prime}=H+H(v-1)=H v,
$$

and so

$$
H=\frac{v^{\prime}}{v} .
$$

By (45), $v$ has finitely many zeros, so we can write

$$
v=P_{1} e^{Q_{1}},
$$

where $P_{1}, Q_{1}$ are polynomials, $P_{1} \not \equiv 0$, and $Q_{1}$ is non-constant because $v$ is transcendental. 
Therefore,

$$
w=\frac{v-1}{L e^{Q}}=\frac{P_{1} e^{Q_{1}}-1}{L e^{Q}} .
$$

Now, we can write this as

$$
w=R_{1} e^{S_{1}}+R_{2} e^{S_{2}},
$$

where $R_{1}=P_{1} / L \not \equiv 0, R_{2}=-1 / L \not \equiv 0$ are rational functions and $S_{1}=Q_{1}-Q, S_{2}=-Q$ are polynomials.

Here, $R_{1} e^{S_{1}}$ and $R_{2} e^{S_{2}}$ are linearly independent because $Q_{1}$ is non-constant. Now, we get

$$
0=w^{\prime \prime}+P w=J_{1} e^{S_{1}}+J_{2} e^{S_{2}}=J_{1} e^{Q_{1}-Q}+J_{2} e^{-Q},
$$

where $J_{1}, J_{2}$ are rational and satisfy

$$
J_{k}=R_{k}^{\prime \prime}+2 R_{k}^{\prime} S_{k}^{\prime}+R_{k}\left(S_{k}^{\prime \prime}+S_{k}^{\prime 2}+P\right) .
$$

Therefore, $J_{1}=J_{2}=0$ because otherwise $e^{Q_{1}}=-J_{2} / J_{1}$ or $e^{-Q_{1}}=J_{1} / J_{2}$. Thus, $R_{1} e^{S_{1}}, R_{2} e^{S_{2}}$ both solve $y^{\prime \prime}+P y=0$ and have finitely many zeros, and they are linearly independent.

Hence, $P$ is constant by [9], which contradicts our assumption in Case (II) that $P$ is nonconstant.

\section{Competing interests}

The author declares that he has no competing interests.

\section{Acknowledgements}

The author would like to thank his supervisor Prof. Jim Langley for his support and guidance. Also, he would like to thank King Abdulaziz University for financial support for his PhD study.

\section{Received: 8 May 2012 Accepted: 17 September 2012 Published: 4 October 2012}

\section{References}

1. Alotaibi, A: On complex oscillation theory. Results Math. 47, 165-175 (2005)

2. Bank, SB, Laine, I: Representations of solutions of periodic second order linear differential equations. J. Reine Angew. Math. 344, 1-21 (1983)

3. Bank, SB, Laine, I, Langley, JK: On the frequency of zeros of solutions of second order linear differential equations. Results Math. 10, 8-24 (1986)

4. Bank, SB, Laine, I, Langley, JK: Oscillation results for solutions of linear differential equations in the complex plane. Results Math. 16, 3-15 (1989)

5. Bank, SB, Langley, JK: Oscillation theory for higher order linear differential equations with entire coefficients. Complex Var. Theory Appl. 16(2-3), 163-175 (1991)

6. Langley, JK: Some oscillation theorems for higher order linear differential equations with entire coefficients of small growth. Results Math. 20(1-2), 517-529 (1991)

7. Langley, JK: On entire solutions of linear differential equations with one dominant coefficient. Analysis 15(2), 187-204 (1995) [Corrections in Analysis 15, 433 (1995)]

8. Rossi, J: Second order differential equations with transcendental coefficients. Proc. Am. Math. Soc. 97, 61-66 (1986)

9. Bank, SB, Laine, I: On the Oscillation theory of $f^{\prime \prime}+A f=0$ where $A$ is entire. Trans. Am. Math. Soc. 273, 351-363 (1982)

10. Hille, E: Ordinary Differential Equations in the Complex Domain. Wiley, New York (1976)

11. Laine, I: Nevanlinna theory and complex differential equations. de Gruyter Studies in Mathematics, vol. 15. de Gruyter, Berlin (1993)

12. Wittich, $\mathrm{H}$ : Neuere Untersuchungen über eindeutige analytische Funktionen. Ergebnisse der Mathematik und ihrer Grenzgebiete, vol. 8. Springer, Berlin (1955)

13. Hayman, WK: Meromorphic Functions. Oxford Mathematical Monographs. Clarendon Press, Oxford (1964)

14. Asiri, A: Common zeros of the solutions of two differential equations. Comput. Methods Funct. Theory 12,67-85 (2012)

15. Asiri, A: Common zeros of the solutions of two differential equations with transcendental coefficients. J. Inequal. Appl. 2011, 134 (2011). doi:10.1186/1029-242X-2011-134 
16. Asiri, A: Common zeros of the solutions of two non-homogeneous first order differential equations. Results Math. (2011). doi:10.1007/s00025-011-0213-y. Published online: 17 November 2011, 1-10

17. Hayman, WK: The local growth of power series: a survey of the Wiman-Valiron method. Can. Math. Bull. 17(3) 317-358 (1974)

doi:10.1186/1029-242X-2012-222

Cite this article as: Asiri: Further results on common zeros of the solutions of two differential equations. Journal of Inequalities and Applications 2012 2012:222.

Submit your manuscript to a SpringerOpen ${ }^{\circ}$ journal and benefit from:

- Convenient online submission

- Rigorous peer review

- Immediate publication on acceptance

Open access: articles freely available online

- High visibility within the field

- Retaining the copyright to your article 